\title{
Diffuse Lewy body disease manifesting as corticobasal syndrome
}

\author{
A rare form of Lewy body disease
}

Koji Kasanuki, MD, PhD, Keith A. Josephs, MD, Tanis J. Ferman, PhD, Melissa E. Murray, PhD, Shunsuke Koga, MD, PhD, Takuya Konno, MD, PhD, Nobutaka Sakae, MD, PhD, Adam Parks, PhD, Ryan J. Uitti, MD, Jay A. Van Gerpen, MD, Neill R. Graff-Radford, MBBS, Zbigniew K. Wszolek, MD, and Dennis W. Dickson, MD

Neurology ${ }^{\circledR}$ 2018;91:e268-e279. doi:10.1212/WNL.0000000000005828

\section{Abstract \\ Objective}

To describe clinical and pathologic characteristics of diffuse Lewy body disease (DLBD) manifesting as corticobasal syndrome (CBS).

\section{Methods}

In 523 autopsy-confirmed cases of DLBD, we identified 11 patients diagnosed with CBS. For comparison, we studied 22 DLBD brains with antemortem presentation of dementia with Lewy bodies (DLB). Given previous studies suggesting the importance of pathology in peri-Rolandic cortices in CBS, we used digital pathology to count Lewy bodies and to quantify intracytoplasmic and neuritic $\alpha$-synuclein and phospho-tau burden in the motor cortex.

\section{Results}

DLBD patients with antemortem features of CBS were significantly younger at disease onset and less likely to have REM sleep behavior disorder than DLBD cases who met clinical criteria for DLB during life. Patients with DLBD manifesting as CBS had more Lewy bodies in the motor cortex than DLBD manifesting as clinically probable DLB. Three cases had concomitant progressive supranuclear palsy and 4 cases had concomitant Alzheimer disease as probable correlates of CBS.

\section{Conclusion}

The neuropathology underlying CBS is heterogeneous, including corticobasal degeneration, Alzheimer disease, and progressive supranuclear palsy. This study suggests that atypical variants of Lewy body disease with severe peri-Rolandic Lewy-related pathology can present clinically as CBS. Patients with DLBD who present as CBS tend to have an earlier age at onset and are less likely to have clinical features of DLB, such as dream enactment behavior during sleep, visual hallucinations, and levodopa-responsive parkinsonism. Future studies with biofluid or molecular imaging biomarkers for $\alpha$-synuclein will permit better recognition of this uncommon pathologic substrate of CBS.

\author{
Correspondence \\ Dr. Dickson \\ dickson.dennis@mayo.edu
}




\section{Glossary}

$\mathrm{AD}=$ Alzheimer disease ATP = Alzheimer-type pathology; $\mathrm{CBD}=$ corticobasal degeneration $\mathbf{C B S}=$ corticobasal syndrome; DLB = dementia with Lewy bodies; DLBD = diffuse Lewy body disease; LBD = Lewy body disease; PSP = progressive supranuclear palsy; RBD = REM sleep behavior disorder.

Corticobasal syndrome (CBS) is characterized by a constellation of signs and symptoms including limb apraxia, cortical sensory loss, myoclonus, and alien limb sign, as well as bradykinesia, dystonia, tremor, and asymmetric rigidity. ${ }^{1,2} \mathrm{Sev}$ eral neuropathologic processes can produce CBS, including Alzheimer disease (AD), corticobasal degeneration (CBD), and progressive supranuclear palsy (PSP). ${ }^{3-6}$ Lewy-related pathology is rarely the underlying substrate of $\mathrm{CBS}^{6-8}$ In the Queen Square brain bank, only 2 Lewy-related Parkinson disease cases presented with CBS, while the majority had tau pathology. ${ }^{6}$ Only 2 published reports have described autopsyconfirmed diffuse Lewy body disease (DLBD) manifesting as CBS. ${ }^{7,8}$ One was a 70 -year-old woman with severe Alzheimer pathology in the motor cortex, raising questions about the significance of Lewy-related pathology to her presentation. ${ }^{8}$ The other report failed to provide information about the density or distribution of neocortical Lewy-related pathology. ${ }^{7}$ Thus, it remains unclear whether DLBD can present with a focal cortical syndrome such as CBS. We addressed this gap in knowledge by reviewing DLBD cases from a brain bank of neurodegenerative disorders at Mayo Clinic. In a subset of 523 cases in which $\alpha$-synuclein immunohistochemistry was used to quantify Lewy-related pathology (cases processed after 1997), we identified 11 cases of DLBD with antemortem CBS: 4 with concomitant $\mathrm{AD}$ and 3 with concomitant PSP that could account for the CBS, but also 4 cases in which DLBD was the probable correlate of the syndrome. We describe demographic features and clinical features of DLBD manifesting as CBS and attempt to understand what neuropathologic features contribute to this distinct syndrome and what clinical features might be helpful in its antemortem recognition.

\section{Methods}

\section{Case selection}

Cases with a neuropathologic diagnosis of $\mathrm{DLBD}^{9,10}$ were from the Mayo Clinic brain bank for neurodegenerative disorders in Jacksonville, FL. From 523 autopsy-confirmed DLBD cases between January 1998 and August 2017, we identified 11 patients with antemortem clinical features consistent with probable CBS, using criteria proposed by Armstrong et al., ${ }^{2}$ during life, in addition to some features suggesting Lewy body disease. For comparison, 22 DLBD cases with clinical features of dementia with Lewy bodies (DLB) were identified from a prospective cohort followed at Mayo Clinic. ${ }^{11}$ DLBD with clinical syndrome of DLB had a range of concomitant Alzheimer-type pathology (ATP), ${ }^{12}$ but none had other major pathologic processes.

\section{Standard protocol approvals, registrations, and patient consents}

Mayo Clinic brain bank operates under procedures approved by the Mayo Clinic institutional review board. All brains were donated after consent from the next-of-kin or an individual with legal authority to grant such permission. The institutional review board has determined that clinicopathologic studies on deidentified postmortem tissue samples are exempt from human subject research.

\section{Clinical assessment}

Clinical information was obtained through a review of medical records (table 1). Data regarding motor features were based on formal neurologic examination as documented by a behavioral neurologist or movement disorder specialist. Cognitive and psychiatric features were based on neuropsychological and psychiatric evaluations, and when available, confirmation of REM sleep behavior disorder (RBD) was based on overnight polysomnography. For each case, presence of clinical symptoms and signs were noted in relationship to the disease course. Age at onset was defined as the presence of the earliest diagnostic feature recorded in clinical visits. Using features from diagnostic criteria, ${ }^{2,13}$ the focus was on the following: (1) CBS clinical features, noting any asymmetry, including limb rigidity, limb dystonia, limb myoclonus, limb apraxia, cortical sensory loss, and alien limb phenomena; (2) DLB clinical features including progressive cognitive decline, fluctuation, visual hallucinations, dream enactment behavior, and features of parkinsonism; and (3) other neurologic signs such as cervical dystonia, pyramidal signs, vertical gaze palsy, axial rigidity, dysphagia, and expressive aphasia.

\section{Tissue sampling and pathologic assessment}

All cases had standardized processing and were evaluated by a single neuropathologist (D.W.D.). Right or left hemi-brains were evaluated in all cases, but most were the left side ( 2 of 11 DLBD manifesting as CBS [CBS-DLBD] and 4 of 22 DLBD manifesting as DLB [DLB-DLBD] were the right side). In addition to histologic evaluation, presence and severity of Alzheimer pathology were assessed with thioflavin $S$ fluorescent microscopy. A Braak neurofibrillary tangle stage ${ }^{14}$ and Thal amyloid phase ${ }^{15}$ were assigned based on lesion counts in cortical and subcortical areas with thioflavin $S$ fluorescent microscopy.

Lewy-related pathology was assessed with immunohistochemistry for $\alpha$-synuclein in 5 regions of neocortex, as well as amygdala, olfactory bulb (if available), basal forebrain, and brainstem. The method used for $\alpha$-synuclein 
Table 1 Demographic and clinical features of DLBD manifesting as CBS compared to DLBD manifesting as DLB

\begin{tabular}{|c|c|c|c|}
\hline Features & CBS-DLBD & DLB-DLBD & $p$ Value \\
\hline Demographics & $\mathrm{n}=11$ & $\mathrm{n}=22$ & \\
\hline Males, n (\%) & $6(55)$ & $18(82)$ & 0.12 \\
\hline Age at onset, y, median (Q1, Q3) & $58(51,64)$ & $71(65,75)$ & 0.005 \\
\hline Age at death, y, median (Q1, Q3) & $65(57,71)$ & $77(72,84)$ & 0.003 \\
\hline Disease duration, $y$, median $(\mathrm{Q} 1, \mathrm{Q} 3)$ & $6(5,7)$ & $8(5,8)$ & 0.36 \\
\hline \multicolumn{4}{|c|}{ Corticobasal syndrome clinical features (Armstrong, ${ }^{2}$ 2013), n (\%) } \\
\hline Limb rigidity & $11(100)$ & $16(73)$ & 0.077 \\
\hline Asymmetric & $8(73)$ & $4(18)$ & 0.005 \\
\hline Limb dystonia & $8(73)$ & $1(5)$ & 0.001 \\
\hline Asymmetric & $7(64)$ & $0(0)$ & $<0.001$ \\
\hline Limb myoclonus & $10(91)$ & $2(9)$ & $<0.001$ \\
\hline Asymmetric & $7(64)$ & $2(9)$ & 0.002 \\
\hline Limb apraxia & $11(100)$ & $0(0)$ & $<0.001$ \\
\hline Asymmetric & $10(91)$ & $0(0)$ & $<0.001$ \\
\hline Cortical sensory deficit & $2(18)$ & $0(0)$ & 0.1 \\
\hline Alien limb phenomena & $3(27)$ & $0(0)$ & 0.03 \\
\hline \multicolumn{4}{|c|}{ Dementia with Lewy bodies clinical features (McKeith, ${ }^{13}$ 2017), n (\%) } \\
\hline Progressive cognitive decline & $11(100)$ & $22(100)$ & - \\
\hline \multicolumn{4}{|l|}{ Core clinical features } \\
\hline Fluctuating cognition and alertness & $6(55)$ & $15(68)$ & 0.47 \\
\hline Recurrent visual hallucinations & $5(45)$ & $16(73)$ & 0.15 \\
\hline Probable REM sleep behavior disorder & $2(18)$ & $16(73)$ & 0.009 \\
\hline \multicolumn{4}{|l|}{ Cardinal features of parkinsonism ${ }^{a}$} \\
\hline Bradykinesia & $11(100)$ & $18(82)$ & 0.28 \\
\hline Rest tremor & $10(91)$ & $7(32)$ & 0.11 \\
\hline \multicolumn{4}{|l|}{ Supportive clinical features } \\
\hline Sensitivity to antipsychotic agents & Noted in 1 case & Noted in 2 cases & - \\
\hline Postural instability & $10(91)$ & $13(59)$ & 0.11 \\
\hline Repeated falls & $8(73)$ & $7(32)$ & 0.06 \\
\hline Hypersomnia & $6(55)$ & $15(68)$ & 0.47 \\
\hline Hyposmia & Noted in 1 case & Not assessed & - \\
\hline Levodopa responsiveness, $\mathrm{n} / \mathrm{n}$ of prescribed cases (\%) & $4 / 6(66)$ & $8 / 11(73)$ & 1.0 \\
\hline \multicolumn{4}{|l|}{ Other neurologic features, $n$ (\%) } \\
\hline Cervical dystonia & $7(64)$ & $6(27)$ & 0.07 \\
\hline Pyramidal signs & $6(55)$ & $0(0)$ & $<0.001$ \\
\hline Vertical gaze palsy & $3(27)$ & $0(0)$ & 0.03 \\
\hline Axial rigidity & $8(73)$ & $1(5)$ & $<0.001$ \\
\hline
\end{tabular}


Table 1 Demographic and clinical features of DLBD manifesting as CBS compared to DLBD manifesting as DLB (continued)

\begin{tabular}{llll}
\hline Features & CBS-DLBD & DLB-DLBD & $\boldsymbol{p}$ Value \\
\hline Dysphagia & $3(27)$ & $0(0)$ & 0.03 \\
\hline Expressive aphasia (not fluctuating) & $3(27)$ & $0(0)$ & 0.03 \\
\hline
\end{tabular}

Abbreviations: CBS-DLBD = diffuse Lewy body disease manifesting as corticobasal syndrome; DLB-DLBD = diffuse Lewy body disease manifesting as dementia with Lewy bodies; $\mathrm{Q}=$ quartile.

For pyramidal signs, 4 abnormal neurologic findings were recorded in DLBD: (1) brisk deep tendon reflexes ( $\geq 3+$ ); (2) pathologic reflexes; (3) clonus; and (4) hypertonicity.

a Limb rigidity, one of the cardinal features of parkinsonism, is listed in corticobasal syndrome clinical features.

immunohistochemistry (rabbit polyclonal [NACP], 1:3,000 with $95 \%$ formic acid pretreatment and Dako EnVision reagents [Carpinteria, $\mathrm{CA}]$ ) gives comparable sensitivity to other methods. ${ }^{16}$ After surveying the entire anatomical region at low power, the field with the highest density of Lewy bodies was counted at $\times 200$ magnification. ${ }^{17}$ Additional characterization included tau immunohistochemistry in motor cortex (mouse monoclonal [CP13], 1:1,000; from Peter Davies, $\mathrm{PhD}$, Feinstein Institute, North Shore Hospital, NY). Immunostained slides were scanned and quantitatively assessed with ImageScope analysis software (Aperio Technologies, Vista, CA). Custom-designed color deconvolution algorithms ${ }^{18}$ were used to detect positive pixels (figure $1, \mathrm{~A}$ and B) based on optical density. For each immunostain, positive pixels were converted to percentage burden of the total region of interest.

\section{Statistical analyses}

The median and interquartile ranges are reported, along with unadjusted $p$ values from a 2 -sided, 2-sample, nonparametric Mann-Whitney rank sum test for continuous variables. Chisquare and Fisher exact tests were used for categorical variables, as appropriate. Correlation of Lewy-related pathology to clinical variables was assessed with the Spearman rank order correlation method. Kruskal-Wallis rank sum tests were used for ordered categorical or continuous variables in pathologic analyses. In pairwise tests, significant $p$ values were set at $p \leq 0.008$. For comparisons of Lewy body counts between DLBD groups, Mann-Whitney rank sum tests were performed and $p \leq 0.005$ using a Bonferroni correction was considered as significant.

\section{Data availability}

This clinicopathologic study is not a clinical trial; therefore, the requirements of the International Committee of Medical Journal Editors have not been applied. We follow the data availability policies of Neurology ${ }^{\circledR}$, and do not report any specific exception. Deidentified data are made available on request.

\section{Results}

\section{Demographics and concomitant pathologies}

The patients with CBS were from of a series of 523 DLBD cases. DLBD cases included $60 \%$ men $(\mathrm{n}=314)$, with an average age at death of $78 \pm 8$ years of age. The average brain weight was 1,140 $\pm 150 \mathrm{~g}$; the median Braak neurofibrillary tangle stage was IV (25th \%-tile: III; 75th \%-tile: V); and the median Thal amyloid phase was 4 (25th \%-tile: 3 ; 75 th \%-tile: $5)$. Most had dementia $(n=429)$, and 281 had dementia with parkinsonism (DLB or Parkinson disease dementia); a smaller subset $(\mathrm{n}=75)$ had primarily parkinsonism. A few $(\mathrm{n}=19)$ had focal cortical syndromes, such as behavioral variant frontotemporal dementia, progressive aphasia, or CBS. Eleven cases fulfilled clinical criteria of probable CBS, ${ }^{2}$ except for variable concurrent DLB features as described below. They had earlier symptomatic disease onset ( 58 years old, $p=0.005)$ and age at death (65 years old, $p=0.003$ ) compared to prospectively evaluated cases of DLBD manifesting as DLB. There was no significant difference regarding disease duration ( 6 years, $p=0.36$ ) or $\operatorname{sex}(55 \%$ men, $p=0.12)$ (table 1). As summarized in table 2, CBS-DLBD pathology could be subclassified into 3 groups depending on concomitant pathology: subgroup A ( $n=4$, "CBS-DLBD + ATP") had DLBD with mild to moderate ATP consistent with intermediate-likelihood $\mathrm{AD} .{ }^{10}$ In these cases, the pathologic process that drives the clinical syndrome is presumed to be DLBD. Subgroup B ( $n=4$, "CBS-DLBD + AD") had DLBD with severe $\mathrm{AD}$ pathology. In these cases, it is reasonable to assume that $\mathrm{AD}$ pathology drives the clinical syndrome, as it is increasingly recognized that $\mathrm{AD}$, particularly atypical forms of $\mathrm{AD}$ with cortical predominant neurofibrillary degeneration (so-called "hippocampal-sparing $\mathrm{AD}^{119}$ ), can present as $\mathrm{CBS}^{20}{ }^{20}$ One of these cases (case 6) had hippocampal-sparing AD. Subgroup C ( $n=3$, "CBS-DLBD + PSP") had DLBD with concomitant pathology of PSP. In this group, it is reasonable to assume that the PSP pathology is the primary factor driving the clinical syndrome, because it is increasingly recognized that atypical PSP can mimic CBS. ${ }^{21,22}$

\section{Clinical characteristics of CBS-DLBD}

Although all 11 patients with CBS-DLBD had neuropathologic findings consistent with "intermediate" to "high" likelihood of DLB, ${ }^{13}$ the initial symptoms were not always compatible with DLB. They were more likely to have clinical features consistent with CBS (table 1), such as asymmetric clumsiness and involuntary hand movements, pyramidal signs, and axial rigidity. Five patients (45\%) were either diagnosed with or suspected to have CBS early in the disease course (shown with footnote designator $\mathrm{c}$ in table 2), but initial diagnoses by neurologists in the other patients included 

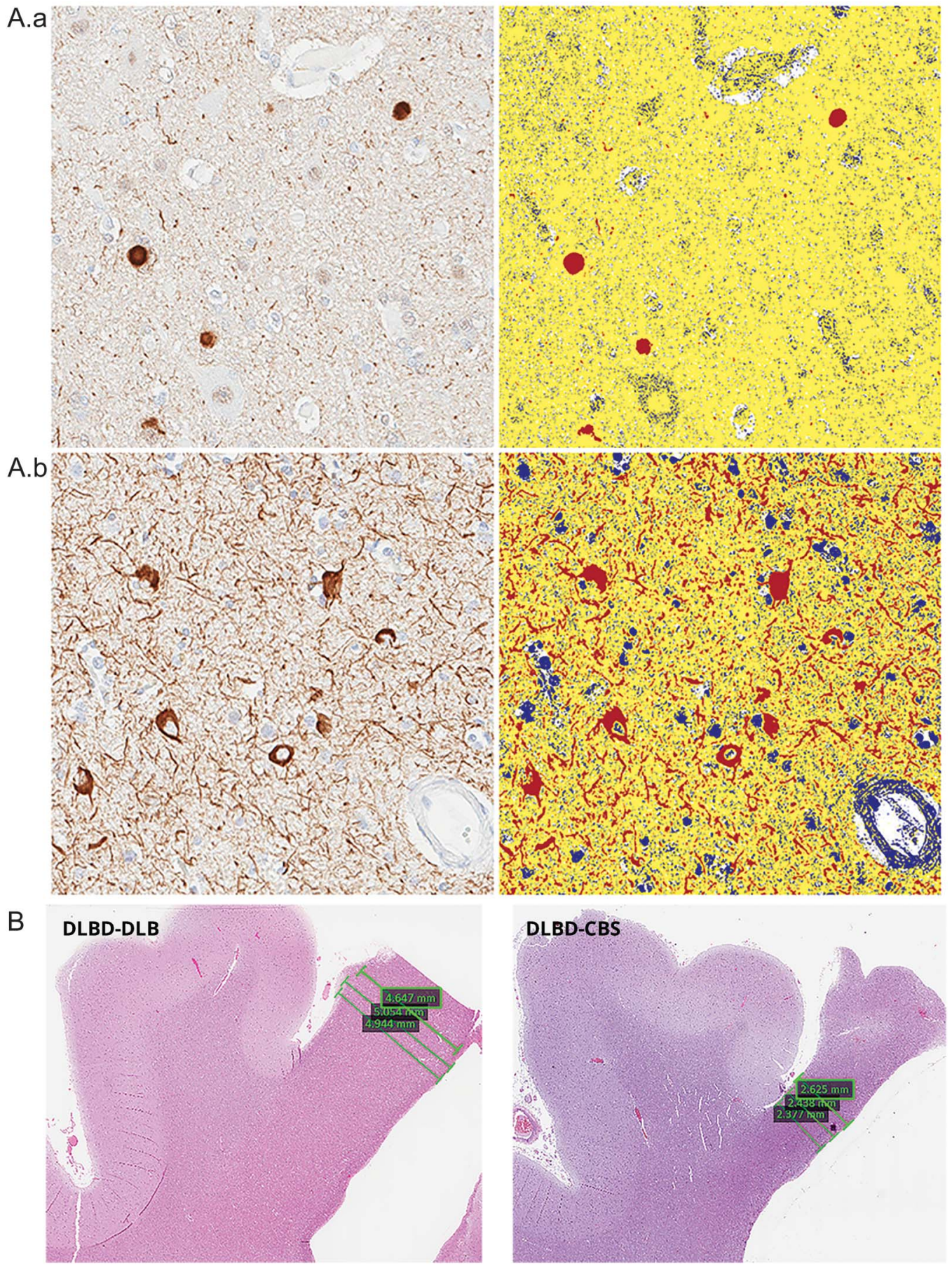

(A.a) a-Synuclein immunohistochemistry (left) and digital pathology mask (right); (A.b) Phospho-tau immunohistochemistry (left) and digital pathology mask (right). For digital pathology, red indicates positive signal. (B) Illustrative example of corpus callosum measurements. ImageScope ruler tool was used to mark the thickness of the anterior corpus callosum in a standardized section at the level of the nucleus accumbens. DLBD-CBS = diffuse Lewy body disease manifesting as corticobasal syndrome; DLBD-DLB = diffuse Lewy body disease manifesting as dementia with Lewy bodies.

$\mathrm{AD}$, Parkinson disease, $\mathrm{DLB}$, and PSP. When considering the constellation of clinical features during the disease course (table 1), all CBS-DLBD had limb rigidity and apraxia, and 10 (91\%) had asymmetric apraxia and myoclonus. In contrast, cortical sensory loss, alien limb phenomenon, and aphasia were less common. Of the clinical features suggestive of DLB, RBD was significantly less frequent in CBS-DLBD $(18 \%, p=$
0.009). Another core feature of DLB, recurrent visual hallucinations not treatment-associated, was relatively low in CBSDLBD (45\%). Unlike CBS with underlying CBD pathology, ${ }^{23,24}$ moderate to good response to levodopa was noted in 4 DLBD with CBS, and 3 had sustained responses. When these features of Lewy body disease are strictly applied as exclusion criteria for probable CBD by Armstrong et al., ${ }^{2} 7$ 
Table 2 Demographics and clinical and pathologic features of DLBD manifesting as CBS

\begin{tabular}{|c|c|c|c|c|c|c|c|c|c|}
\hline Case & $\begin{array}{l}\text { Other } \\
\text { pathologies }\end{array}$ & Sex & $\begin{array}{l}\text { Age at } \\
\text { onset, } y\end{array}$ & $\begin{array}{l}\text { Age at } \\
\text { death, y }\end{array}$ & $\begin{array}{l}\text { Disease } \\
\text { duration, } \\
\text { y }\end{array}$ & Initial Diagnosis & $\begin{array}{l}\text { Initial signs and } \\
\text { symptoms }\end{array}$ & $\begin{array}{l}\text { Final } \\
\text { Dx }\end{array}$ & CDLB \\
\hline \multicolumn{10}{|c|}{ Overall: CBS-DLBD $(n=11)$} \\
\hline \multicolumn{10}{|c|}{ Subgroup A: DLBD + ATP } \\
\hline 1 & $-^{a}$ & M & 46 & 54 & 8 & $\begin{array}{l}\text { Dementia vs depressive } \\
\text { pseudodementia }\end{array}$ & $\begin{array}{l}\text { Slow speech, } \\
\text { depression, anxiety }\end{array}$ & $\mathrm{CBS}^{\mathrm{b}}$ & High \\
\hline $2^{c}$ & $-^{a}$ & $\mathrm{~F}$ & 58 & 67 & 9 & CBS & $\begin{array}{l}\text { Asymmetric } \\
\text { clumsiness, dyscalculia }\end{array}$ & $\mathrm{CBS}^{\mathrm{b}}$ & High \\
\hline $3^{c}$ & $-{ }^{a}$ & $\mathrm{~F}$ & 56 & 59 & 3 & CBS & $\begin{array}{l}\text { Asymmetric jerkiness, } \\
\text { asymmetric } \\
\text { clumsiness, acalculia }\end{array}$ & CBS & High \\
\hline 4 & $-{ }^{d}$ & M & 63 & 69 & 6 & DLB & $\begin{array}{l}\text { Forgetfulness, } \\
\text { distractibility, } \\
\text { fluctuation }\end{array}$ & $\mathrm{CBS}^{\mathrm{b}}$ & Interm \\
\hline
\end{tabular}

\begin{tabular}{|c|c|c|c|c|c|c|c|c|c|}
\hline \multicolumn{10}{|c|}{ Subgroup B: DLBD + AD } \\
\hline 5 & $A D$ & M & 64 & 71 & 7 & PD & $\begin{array}{l}\text { Resting tremor, } \\
\text { dysphoria }\end{array}$ & CBS & Interm. \\
\hline 6 & $\mathrm{AD}(\mathrm{HpSp})$ & M & 48 & 53 & 5 & PD & Resting tremor & $\mathrm{CBS}^{\mathrm{b}}$ & Interm. \\
\hline 7 & $A D$ & M & 51 & 57 & 6 & CBS & $\begin{array}{l}\text { Involuntary jerking } \\
\text { movements, speech } \\
\text { difficulty, dysgraphia, } \\
\text { anosmia }\end{array}$ & CBS & Interm. \\
\hline $8^{c}$ & $A D(P C A)$ & $\mathrm{F}$ & 58 & 65 & 7 & CBS & $\begin{array}{l}\text { Involuntary movement } \\
\text { of left hand, dressing } \\
\text { apraxia }\end{array}$ & CBS & Interm. \\
\hline
\end{tabular}

\begin{tabular}{|c|c|c|c|c|c|c|c|c|c|}
\hline \multicolumn{10}{|c|}{ Subgroup C: DLBD + PSP } \\
\hline 9 & PSPa & $\mathrm{F}$ & 80 & 86 & 6 & $A D$ vs $D L B$ & $\begin{array}{l}\text { Memory impairment, } \\
\text { shuffling gait, agitation }\end{array}$ & $\mathrm{CBS}^{\mathrm{b}}$ & High \\
\hline $10^{c}$ & PSP $^{d}$ & M & 77 & 82 & 5 & CBS & $\begin{array}{l}\text { Asymmetric } \\
\text { clumsiness, aphasia, } \\
\text { fall, agraphesthesia }\end{array}$ & $\mathrm{CBS}^{\mathrm{b}}$ & Interm. \\
\hline 11 & $\mathrm{PSP}^{\mathrm{a}}$ & $\mathrm{F}$ & 53 & 60 & 7 & CBS vs PD vs PSP & $\begin{array}{l}\text { Asymmetric } \\
\text { bradykinesia, mild } \\
\text { resting tremor, } \\
\text { bradyphrenia, fatigue }\end{array}$ & $\mathrm{CBS}^{\mathrm{b}}$ & High \\
\hline \multicolumn{10}{|c|}{ Comparison group: DLB-DLBD $(n=22)$} \\
\hline 12 to $33^{c}$ & $\begin{array}{l}\text { AD in } 6 \\
\text { cases }\end{array}$ & $\begin{array}{l}\text { Male: } \\
55 \%\end{array}$ & $\begin{array}{l}\text { Median: } \\
65\end{array}$ & $\begin{array}{l}\text { Median: } \\
77\end{array}$ & Median: 8 & $\begin{array}{l}\text { Depression, DLB, mild } \\
\text { dementia, } \mathrm{MCl}, \mathrm{MCl} \text { due to } \\
\text { DLB, OCD due to DLB, PD, } \\
\text { PD-MCI }\end{array}$ & $\begin{array}{l}\text { None had clinical } \\
\text { features suggestive of } \\
\text { CBS }\end{array}$ & $\begin{array}{l}\text { All } \\
\text { DLB }\end{array}$ & $\begin{array}{l}\text { Interm.: } \\
\text { high, 16: } \\
6\end{array}$ \\
\hline
\end{tabular}

Abbreviations: $A D=$ Alzheimer disease refers to cases with senile plaques and neurofibrillary tangles consistent with high-likelihood $A D^{10} ; A T P=A l z h e i m e r-$

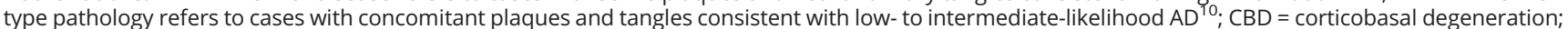
CBS-DLBD = diffuse Lewy body disease manifesting as corticobasal syndrome; CDLB likelihood = assessment of the likelihood that the pathologic findings would be associated with the clinical syndrome of dementia with Lewy bodies according to the Fourth Consensus Report of the DLB Consortium ${ }^{13}$; DLB = dementia with Lewy bodies; $D L B D=$ diffuse Lewy body disease; $D L B D+A D=D L B D$ with severe concomitant $A D$ pathology manifesting as CBS; DLBD + ATP = DLBD with mild to moderate concomitant Alzheimer-type pathology manifesting as CBS; DLB-DLBD = DLBD manifesting as DLB; DLBD + PSP = DLBD with concomitant progressive supranuclear palsy pathology manifesting as CBS; $\mathrm{Dx}=$ diagnosis; $\mathrm{HpSp}=$ hippocampal-sparing $\mathrm{AD} ;$ Interm. = intermediate; $\mathrm{MCl}=$ mild cognitive impairment; $\mathrm{OCD}=$ obsessive compulsive disorder; $\mathrm{PCA}=\mathrm{AD}$ with posterior cortical atrophy; $\mathrm{PD}=\mathrm{Parkinson}$ disease; $\mathrm{PD}-\mathrm{MCl}=\mathrm{MCl}$ due to $\mathrm{PD}$; PSP = progressive supranuclear palsy.

In 11 DLBD manifesting as CBS (CBS-DLBD) cases, there were 2 types of clinical course: cases presenting with early signs and symptoms of CBS (cases 2, 3, 7, 8, and 10) and cases converting to CBS during the disease course (cases 1,4,5,6, 9, and 11). None of the 22 DLBD manifesting as DLB cases had features of CBS. In CBS-DLBD, when features of Lewy body parkinsonism (e.g., resting tremor, excellent and sustained response to L-dopa or hallucinations) are strictly applied as exclusion criteria for probable CBD, ${ }^{2}$ then 7 of 11 (64\%) CBS-DLBD cases failed to meet research CBD criteria (i.e., "CBS ${ }^{\mathrm{b} \text { ") }}$

${ }^{a}$ ATP refers to cases with plaques and tangles consistent with low-likelihood AD. ${ }^{10}$

${ }^{b}$ DLBD manifesting as CBS, but also fulfilling exclusion criteria of probable sporadic CBD ${ }^{2}$ when considering Lewy body parkinsonism features.

${ }^{c}$ Five patients $(45 \%)$ were either diagnosed with or suspected to have CBS early in the disease course.

${ }^{d}$ ATP refers to cases with plaques and tangles consistent with intermediate-likelihood AD. ${ }^{10}$ 
of 11 CBS-DLBD cases (64\%) did not fit this diagnosis (i.e., "CBS" in table 2). Vertical gaze palsy, a characteristic sign of PSP, was noted in 3 DLBD manifesting as CBS, only one of whom had concomitant PSP pathology. Global cognitive deterioration, including memory impairment, was observed in all patients. Behavioral and neuropsychiatric problems were variable and did not differ between the DLBD groups.

\section{Neuropathologic findings}

Neuropathologic characteristics are summarized in table 3. The brain weight in CBS-DLBD tended to be less than that in DLB-DLBD $(p=0.052)$. Disproportionate atrophy in convexity frontal and parietal cortices, including atrophy in premotor and motor cortices, was noted in CBS-DLBD (figure 2, $\mathrm{C}$ and $\mathrm{D}$ ). The corpus callosum at the level of the anterior cingulate gyrus was significantly thinner in CBS-DLBD ( $p=$ 0.026, figure 1B) than that in DLB-DLBD. This observation was confirmed in one patient on antemortem MRI (table 1 and figure $2 \mathrm{~B}$ ). Using pathologic criteria from the fourth report of the Dementia with Lewy Bodies Consortium, ${ }^{13} 5$ CBS-DLBD (45\%) and 16 DLB-DLBD (73\%) were classified as high-likelihood DLB. The amount of ATP as indicated by Braak neurofibrillary tangle stage and Thal amyloid phase was not significantly different between CBS-DLBD and DLBDLBD. Although $\alpha$-synuclein burden in the motor cortex did not reach statistical significance, the number of Lewy bodies was highly correlated with $\alpha$-synuclein burden (Spearman $\rho=$ $\left.0.96,2.0 \times 10^{-7}\right)$ and was significantly greater in CBS-DLBD (median $7, p<0.001$ ) than in DLB-DLBD (median 1). Both tau burden and spongiosis were significantly greater in CBSDLBD. Neuropathologic findings in CBS-DLBD subgroups are illustrated in figures 2 and 3. Compared with DLB-DLBD, subgroup A (CBS-DLBD + ATP) had more cortical Lewy bodies (median 14, $p=0.004$ ) and greater $\alpha$-synuclein burden (median $0.30 \%, p=0.025$ ). In contrast, subgroup B (CBS-

Table 3 Neuropathologic characteristics of DLBD manifesting as CBS and of DLBD manifesting as DLB

\begin{tabular}{|c|c|c|c|}
\hline & CBS-DLBD $(n=11)$ & DLB-DLBD $(n=22)$ & $p$ Value \\
\hline Brain weight, g, mean (SD) & $1,124(126)$ & $1,221(133)$ & 0.052 \\
\hline Thickness of corpus callosum, mm, median (Q1, Q3) & $3.7(3.2,4.7)$ & $4.6(4.2,5.1)$ & 0.026 \\
\hline DLB likelihood (CDLB), intermediate/high & $6 / 5$ & $6 / 16$ & 0.15 \\
\hline Braak NFT stage, median (Q1, Q3) & $\mathrm{V}(\mathrm{IV}, \mathrm{V})$ & IV $(I V, V)$ & 0.26 \\
\hline Thal amyloid phase, median (Q1, Q3) & $4(4,5)$ & $5(4,5)$ & 0.52 \\
\hline \multicolumn{4}{|l|}{ Motor cortex pathologies, median (Q1, Q3) } \\
\hline 1. Lewy body counts & $7(1,13)$ & $1(0,2)$ & $<0.001$ \\
\hline 2. Synuclein burden, $\%$ & $0.1(0.03,0.3)$ & $0.05(0.02,0.1)$ & 0.11 \\
\hline 3. Tau burden, $\%$ & $0.83(0.22,3.5)$ & $0.02(0.009,0.31)$ & 0.003 \\
\hline 4. Spongiosis score & $0(0,1)$ & $0(0,0)$ & 0.002 \\
\hline 5. Betz cell neuronal loss score & $0(0,0)$ & $0(0,0)$ & 1.0 \\
\hline \multicolumn{4}{|l|}{ Lewy body counts in other cortices, median (Q1, Q3) } \\
\hline Parahippocampal gyrus & $25(16,27)$ & $25(15,26)$ & 0.79 \\
\hline Anterior cingulate gyrus & $17(12,28)$ & $16(12,17)$ & 0.18 \\
\hline Superior frontal gyrus & $22(10,31)$ & $8(6,14)$ & 0.007 \\
\hline Middle frontal gyrus & $12(7,20)$ & $6(4,11)$ & 0.017 \\
\hline Superior temporal gyrus & $18(12,25)$ & $16(9,21)$ & 0.18 \\
\hline Inferior parietal lobule & $9(5,20)$ & $5(3,8)$ & 0.05 \\
\hline \multicolumn{4}{|l|}{ Neuronal loss scores in brainstem, median (Q1, Q3) } \\
\hline Substantia nigra (midbrain) & $2(2,3)$ & $2(2,3)$ & 0.64 \\
\hline Locus ceruleus (pons) & $1(1,2)$ & $3(1,3)$ & 0.016 \\
\hline Dorsal motor nucleus of vagus (medulla) & $3(1,3)$ & $2(1,3)$ & 0.39 \\
\hline
\end{tabular}

Abbreviations: CBS-DLBD = diffuse Lewy body disease manifesting as corticobasal syndrome; DLB-DLBD = diffuse Lewy body disease manifesting as dementia with Lewy bodies; DLB likelihood (CDLB) = assessment of the likelihood that the pathologic findings would be associated with the clinical syndrome of DLB according to the Fourth Consensus Report of the Dementia with Lewy Bodies Consortium ${ }^{13}$; NFT = neurofibrillary tangle; Q = quartile. 

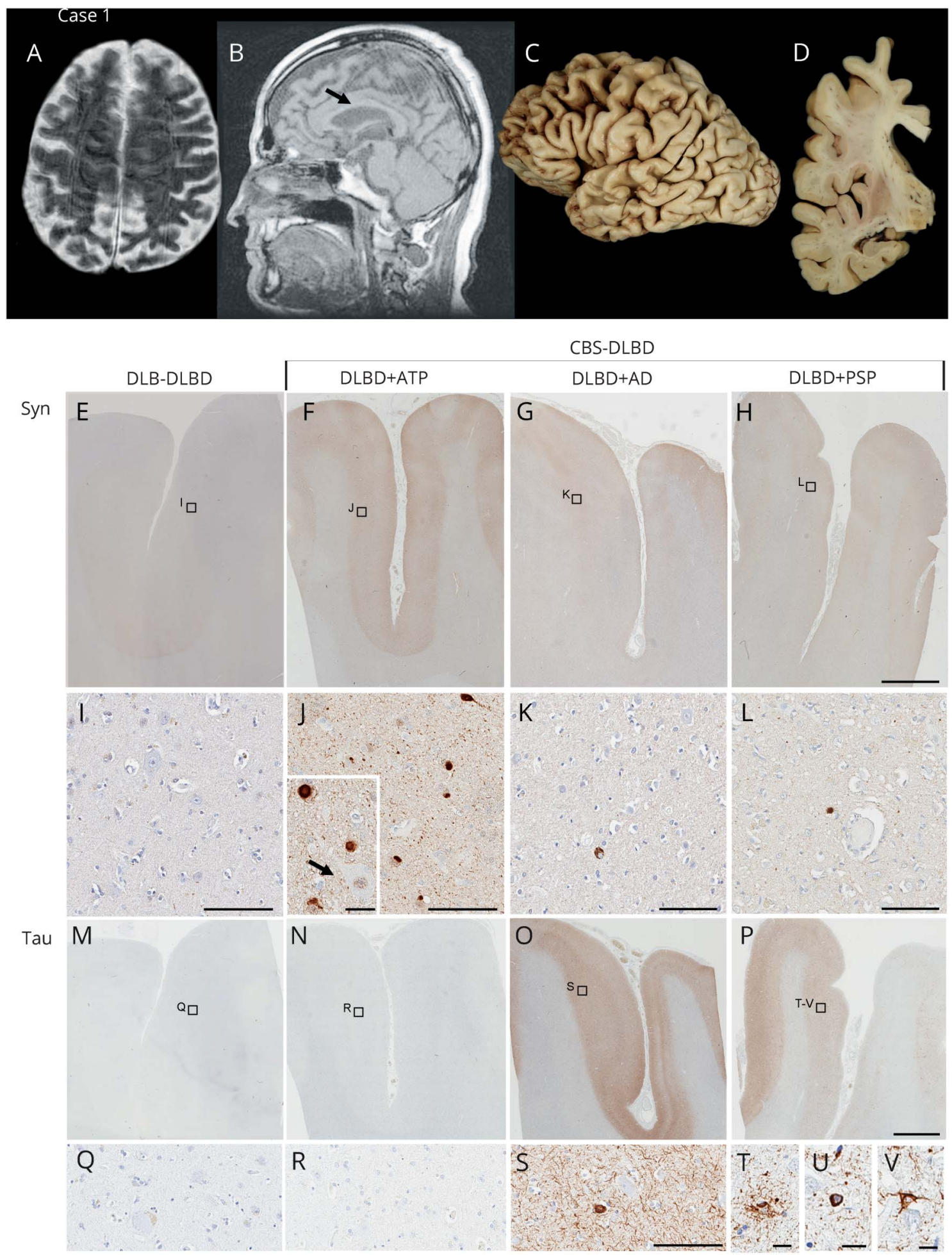

(A, B) Antemortem MRI (T1) of DLBD manifesting as CBS (case 1 in table 1). Note widening of frontoparietal sulci and thinning of the corpus callosum. (C, D) Macroscopic images show disproportionate cortical atrophy in frontoparietal cortices, including peri-Rolandic and premotor cortices. (E-V) Immunohistochemistry of motor cortex. $a-S y n u c l e i n(E-L)$ and phospho-tau $(M-V)$. In DLBD manifesting as CBS, $a$-synuclein pathology is more severe $(F, J)$ than in DLBD manifesting as DLB (E, G-I, K-L). Lewy-related pathology (including cortical Lewy bodies and Lewy neurites) is marked in lower cortical layers (layers IV-V). ()). Lewy bodies adjacent to a Betz cell (arrow in ], inset). The numbers of Lewy bodies in CBS with DLBD + AD (K, arrow), as well as CBS with DLBD + PSP (L, arrow) are less than in CBS with DLBD + ATP (see F and J). Tau pathology is less in CBS with DLBD + ATP (N, R) than in CBS with DLBD + AD $(O, S)$ and in CBS with DLBD + PSP $(P, T-V)$. In CBS with DLBD + PSP note presence of typical tau lesions of PSP (tufted astrocyte [T], coiled body [U], and pretangle [V]). Bars: $E-H, M-P=3$ mm; $\mathrm{L}-\mathrm{L}, \mathrm{Q}-\mathrm{S}=100 \mu \mathrm{m}$; I inset, $\mathrm{T}-\mathrm{V}=25 \mu \mathrm{m}$. CBS-DLBD = diffuse Lewy body disease manifesting as corticobasal syndrome; $\mathrm{DLBD}+\mathrm{AD}=$ diffuse Lewy body disease with severe Alzheimer-type pathology; DLBD + ATP = diffuse Lewy body disease with mild to moderate Alzheimer-type pathology; DLB-DLBD = diffuse Lewy body disease manifesting as typical dementia with Lewy bodies; DLBD + PSP = diffuse Lewy body disease with concomitant pathology of progressive supranuclear palsy; Syn = a-synuclein; Tau = phospho-tau 


\section{Synuclein and tau pathologies in the primary motor cortex}

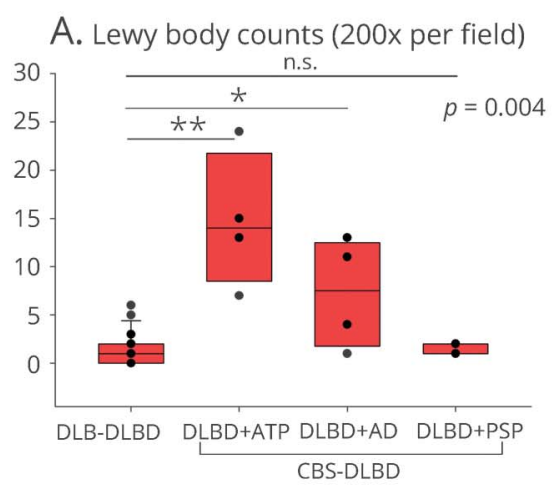

D. Primary motor cortex and parahippocampus

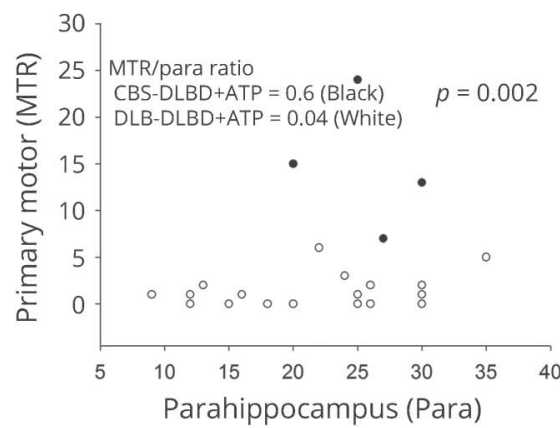

B. Synuclein immunoreactivity (\%)

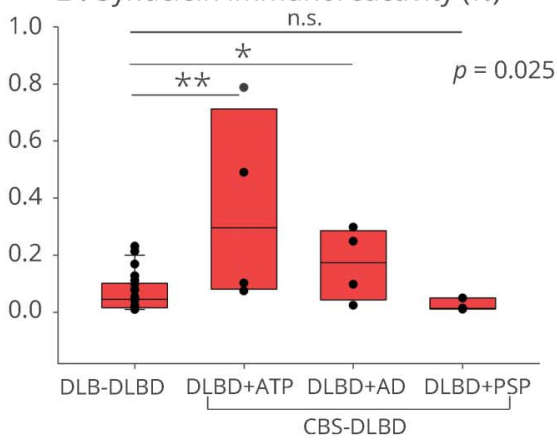

Lewy counts distribution

E. Superior temporal cortex and parahippocampus

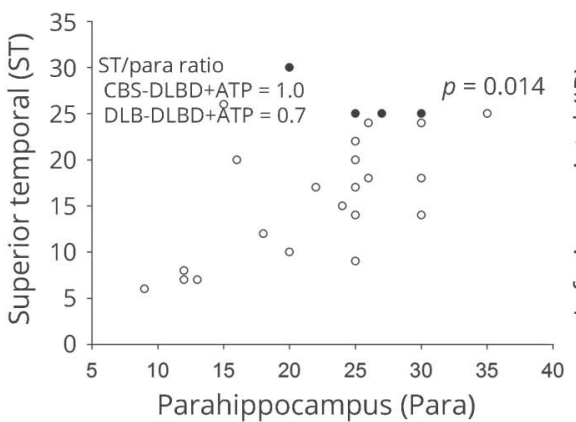

C. Tau immunoreactivity (\%)

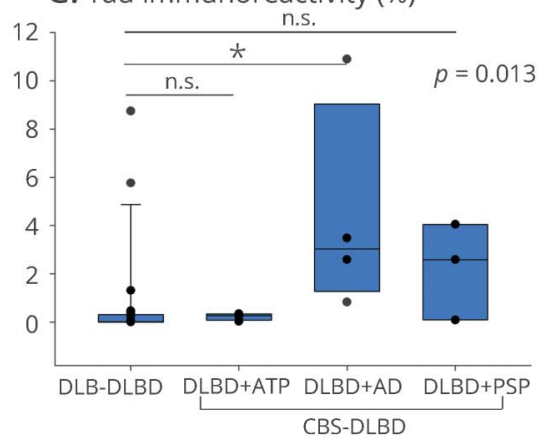

F. Inferior parietal cortex and parahippocampus

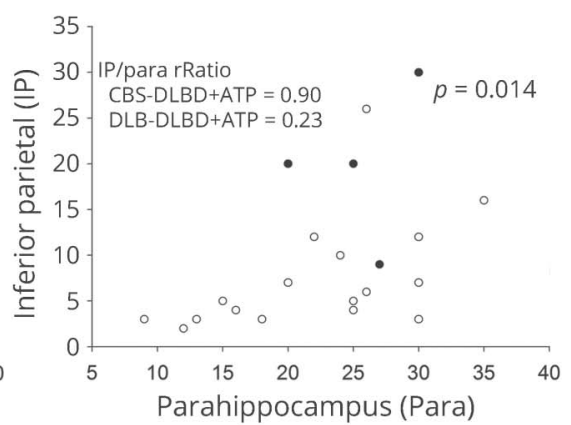

(A, B) Cases of CBS-DLBD + ATP and those with DLBD + AD have more Lewy bodies and greater a-synuclein burden than DLBD manifesting as DLB. (C) Cases of CBS with DLBD + AD have significantly more tau pathology than DLBD manifesting as DLB. The $p$ values represent Kruskal-Wallis rank sum tests. * $p<0.05$ in pairwise tests; ${ }^{* *} p<0.008$ in pairwise tests. (D-F) Lewy body distribution pattern in neocortices and parahippocampal gyrus. (D) Motor cortex and parahippocampal gyrus. (E) Superior temporal cortex and parahippocampal gyrus. (F) Inferior parietal cortex and parahippocampal gyrus. Black circles = CBSDLBD group; white circles = DLB-DLBD group. Compared with nonmotor neocortices, the number of Lewy bodies in the motor cortex is disproportionally higher in CBS-DLBD. The $p$ value represents results of Mann-Whitney rank sum tests. CBS-DLBD = diffuse Lewy body disease manifesting as corticobasal syndrome; DLBD + AD = diffuse Lewy body disease with severe Alzheimer-type pathology; DLBD + ATP = diffuse Lewy body disease with mild to moderate Alzheimer-type pathology; DLB-DLBD = diffuse Lewy body disease manifesting as typical dementia with Lewy bodies; DLBD + PSP = diffuse Lewy body disease with concomitant pathology of progressive supranuclear palsy; n.s. = not significant.

$\mathrm{DLBD}+\mathrm{AD})($ median $3.0 \%)$ and subgroup C (CBS-DLBD + PSP) (median 2.6\%) had greater cortical tau burden than subgroup A (median $0.27 \%$ ). Cortical tau burden in subgroup A was similar to DLB-DLBD (median $0.02 \%, p=0.20$ in pairwise test). CBS-DLBD had more cortical Lewy bodies in superior temporal $(p=0.007)$ and middle frontal $(p=0.017)$ cortices than DLB-DLBD (table 3). The density of Lewyrelated pathology in the motor cortex relative to the density in the limbic lobe (e.g., parahippocampal gyrus) was disproportionally higher in CBS-DLBD than in DLB-DLBD (figure 3D). However, the relative density of Lewy-related pathology in other neocortices was similar between the 2 groups (figure 3, E and F).

\section{Discussion}

The neuropathologic underpinnings of CBS are heterogeneous; the most common findings include CBD, PSP, and $\mathrm{AD}^{3,6,22}$ In this report, we highlight the fact that, while rare,
DLBD can also be found in patients with CBS, and in cases with only mild to moderate concomitant Alzheimer or tau pathology, atypical cortical Lewy-related pathology may be the best neuropathologic correlate for the syndrome. The 4 cases with CBS were from a series of 523 cases of DLBD, suggesting a frequency of approximately $0.8 \%$. Given the referral bias of our brain bank, which is the brain bank for CurePSP, ${ }^{25}$ this is certainly an overestimate.

Of the 11 cases of CBS-DLBD, 7 had other pathologic processes ( $\mathrm{AD}$ or PSP) that might well be the primary correlate of the clinical syndrome, but 4 cases had DLBD with only mild to moderate ATP and no other pathologic explanation for CBS. These cases had 14-fold more Lewy bodies and 6-fold greater $\alpha$-synuclein burden in the motor cortex than DLBDLBD cases. It is rare to find neuropathologic evidence that atypical distribution of Lewy-related pathology, with severe pathology in motor cortex, can present with CBS. A previous case report of CBS-DLBD by Horoupian and Wasserstein ${ }^{8}$ emphasized the importance of pathology in the peri-Rolandic 
cortices in CBS, with focus on the motor cortex, but their patient had severe ATP in the motor cortex, not severe Lewyrelated pathology as in our cases.

Recognizing the nature of underlying pathologic processes in patients with CBS is challenging and especially so for those with DLBD, since there are no biofluid or neuroimaging biomarkers for $\alpha$-synuclein pathology. Of the clinical manifestations noted in our cases, those consistent with CBS lack specificity and are comparable to those observed in CBS due to other pathologic processes. ${ }^{6,26}$ Possible clues to underlying Lewy-related pathology in CBS are features often found in DLB, such as dream enactment behavior consistent with $\mathrm{RBD}$, visual hallucinations, fluctuations in consciousness, systematized delusions, and anosmia (table 1). While it is difficult to generalize from such small numbers, the relatively low frequency of RBD in CBS-DLBD might be attributable to the number of women ( 5 of 11 ) in our cohort, given that RBD is less frequent in women. ${ }^{27}$ The relatively good responsiveness to levodopa, as well as hypersensitivity to dopaminergic agonists, also might be clues to Lewy-related pathology, which contrasts with low frequency of RBD and poor levodopa responsiveness in a clinicopathologic study of CBD. ${ }^{23,24}$ It is worth noting that both pyramidal signs and axial rigidity were common in CBS-DLBD cases. Across subtypes of CBS-DLBD, all 3 DLBD + PSP cases had both of these signs. However, these signs were only seen in $50 \%$ to $75 \%$ of DLBD $+\mathrm{AD}$ and DLBD + ATP cases, suggesting the potentially strong contribution of concomitant PSP pathology for these signs in DLBD.

It remains to be determined whether neuroimaging can be helpful in differentiating various possible pathologic processes that might give rise to the CBS. Molecular imaging holds significant promise for refining antemortem clinical diagnoses. Amyloid imaging combined with MRI has been used to differentiate $\mathrm{CBS}$ due to $\mathrm{AD}$ pathology from $\mathrm{CBS}$ due to $C B D .^{28}$ It is uncertain whether this approach would help differentiate CBS due to DLBD from CBD since almost all patients with DLBD have cortical amyloid, including many with levels comparable to those observed in AD. ${ }^{29}$ Tau imaging may be a useful tool to detect CBS due to tauopathy including $\mathrm{AD}$ and $4 \mathrm{R}$ tauopathies. ${ }^{30-32} \mathrm{At}$ present, there are no molecular ligands for molecular imaging of cortical $\alpha$-synuclein pathology, but this may improve antemortem diagnosis as part of a multimodality diagnostic approach.

Neurobiological basis of CBS is not fully understood; however, involvement of both frontal and parietal multimodal association cortices is consistently noted. Pathology in the supplementary motor area is considered important for limb apraxia, ${ }^{33}$ while pathology in peri-Rolandic somatosensory cortices contributes to cortical sensory loss and peri-Rolandic motor cortices to pyramidal signs. ${ }^{34}$ Our finding of high burden of Lewy-related pathology in the motor cortex is in line with the neuroanatomy of CBS.
The motor and sensory cortices are relatively spared in $\mathrm{AD}$ and Parkinson disease, ${ }^{35}$ and in the present study, there was minimal Lewy-related pathology in the motor cortex in DLBDLBD (table 3). Consequently, routine neuropathologic assessment of motor cortex is not usually required in neuropathologic evaluations of these disorders or for DLBD. ${ }^{13}$ In the present study, $45 \%$ of CBS-DLBD cases were classified as "high-likelihood" DLB, although their antemortem presentations were more characteristic of CBS; therefore, our findings indicate the importance of evaluating peri-Rolandic cortices in cases of DLBD with atypical clinical presentations. Pathology in limbic and neocortical areas were similar in CBSDLBD to those in DLB-DLBD, which suggests that the atypical clinical presentation is not merely due to more severe, but rather an atypical distribution of Lewy-related pathology. An analogous situation has been observed in $\mathrm{AD}$ manifesting as focal cortical syndromes ${ }^{19}$ and suggests that patterns of lesion distribution may define distinct clinicopathologic variants.

A limitation of the present study is that the CBS cases were derived from a retrospective clinicopathologic series of brain bank cases. As such, available clinical information is variable and not systematically collected. Nevertheless, sufficient clinical information was available from neurologists and movement disorder specialists to confidently confirm CBS. In contrast, the comparison group (DLBD manifesting as DLB) was derived from a prospective cohort of DLB cases. Atypical DLBD cases manifesting as CBS would be excluded from this longitudinal cohort. While it is unlikely to be frequent, DLBD manifesting as CBS may go undetected without autopsy. As the brain bank for CurePSP, ${ }^{25}$ we have the opportunity of evaluating a large number of patients with antemortem CBS, and less than half have had CBD pathology at autopsy, similar to findings of the Queen Square brain bank. ${ }^{6}$ A large population-based autopsy study is needed to resolve these issues.

In the current study, we highlight a rare clinical presentation of DLBD and provide evidence that CBS may be linked to atypical distribution of cortical Lewy-related pathology. Our findings suggest that in addition to Alzheimer and frontotemporal degenerative pathologies, Lewy-related pathology may contribute to neuropathologic heterogeneity of CBS.

\section{Author contributions}

K.K.: conceptualization and design of the study, data acquisition, analysis and interpretation of the data, drafted and revised the manuscript. K.A.J.: conceptualization and design of the study, data acquisition, revised the manuscript. T.J.F.: data acquisition, interpretation of the data, revised the manuscript. M.E.M.: conceptualization and design of the study, data acquisition, analysis of the data, revised the manuscript. S.K.: interpretation of the data, revised the manuscript. T.K.: interpretation of the data, revised the manuscript. N.S.: revised the manuscript. A.P.: interpretation of the data, revised the manuscript. R.J.U.: data acquisition, revised the 
manuscript. J.A.V.G.: conceptualization and design of the study, revised the manuscript. N.R.G.-R.: data acquisition, revised the manuscript. Z.K.W.: data acquisition, revised the manuscript. D.W.D.: conceptualization and design of the study, data acquisition, analysis and interpretation of the data, drafted and revised the manuscript.

\section{Acknowledgment}

The authors thank all patients and their families for support of this research. The authors also acknowledge the continuous commitment and teamwork offered by Monica Castanedes Casey, Linda Rousseau, and Virginia Phillips.

\section{Study funding}

Supported by Mayo Clinic Alzheimer's Disease Research Center (P50 AG16574); Udall Center of Excellence for Parkinson's Disease Research (P50 NS72187); Mangurian Foundation Lewy Body Dementia Program at Mayo Clinic; CurePSP; and The Tau Consortium. This study was supported by NIH (P50 NS072187, P50 AG016574, U54 NS100693), the Michael J. Fox Foundation, the Mayo Clinic Alzheimer's Disease and Related Dementias Genetics Program, and by the Mangurian Foundation Lewy Body Dementia Program at Mayo Clinic.

\section{Disclosure}

K. Kasanuki reports no disclosures relevant to the manuscript. K. Josephs is funded by NIH grants R01 AG037491 (principal investigator [PI]); R01 NS89757 (PI); and R21 NS094684 (PI). T. Ferman receives research support from the NIH (P50-AG016574) and from the Mangurian Foundation Lewy Body Dementia Program at Mayo Clinic. M. Murray, S. Koga, T. Konno, N. Sakae, and A. Parks report no disclosures relevant to the manuscript. R. Uitti receives research support by the NIH//NINDS (R01-NS057567), research funding from Advanced Neuromodulation Systems, Inc./St. Jude Medical, and a gift from Carl Edward Bolch, Jr., and Susan Bass Bolch. Dr. Uitti is an editorial board member of Neurology. J. Van Gerpen reports no disclosures relevant to the manuscript. N. Graff-Radford serves on a scientific advisory board for Codman; serves on the editorial boards of The Neurologist and Alzheimer's Research of Therapy; has received publishing royalties from UpToDate, Inc.; and receives research support from Biogen, Lilly, and Axovant. He has consulted for Cytox. Z. Wszolek holds and has contractual rights for receipt of future royalty payments from patents re: A novel polynucleotide involved in heritable Parkinson's disease; receives royalties from editing Parkinsonism and Related Disorders (Elsevier, 2015, 2016) and the European Journal of Neurology (Wiley-Blackwell, 2015, 2016). Dr. Wszolek is partially supported by the NIH/NINDS P50 NS072187, NIH/NIA (primary), and NIH/NINDS (secondary) U01AG045390; Mayo Clinic Center for Regenerative Medicine, Mayo Clinic Center for Individualized Medicine, Mayo Clinic Neuroscience Focused Research Team (Cecilia and Dan Carmichael Family Foundation, and the James C. and Sarah K. Kennedy Fund for Neurodegenerative Disease Research at Mayo Clinic in Florida); the gift from Carl Edward Bolch, Jr., and Susan Bass Bolch, The Sol Goldman Charitable Trust, and Donald G. and Jodi P. Heeringa. D. Dickson receives research support from the NIH (P50-AG016574; P50-NS072187; P01AG003949), from the Mangurian Foundation Lewy Body Dementia Program at Mayo Clinic, and the Robert E. Jacoby Professorship. Dr. Dickson is an editorial board member of Acta Neuropathologica, Annals of Neurology, Brain, Brain Pathology, and Neuropathology, and he is editor in chief of American Journal of Neurodegenerative Disease. Go to Neurology.org/ $\mathrm{N}$ for full disclosures.

Received September 17, 2017. Accepted in final form April 13, 2018.

\section{References}

1. Gibb WR, Luthert PJ, Marsden CD. Corticobasal degeneration. Brain 1989;112: 1171-1192.

2. Armstrong MJ, Litvan I, Lang AE, et al. Criteria for the diagnosis of corticobasal degeneration. Neurology 2013;80:496-503.

3. Boeve BF, Maraganore DM, Parisi JE, et al. Pathologic heterogeneity in clinically diagnosed corticobasal degeneration. Neurology 1999;53:795-800.

4. Ouchi H, Toyoshima Y, Tada M, et al. Pathology and sensitivity of current clinical criteria in corticobasal syndrome. Mov Disord 2014;29:238-244.

5. Josephs KA, Petersen RC, Knopman DS, et al. Clinicopathologic analysis of frontotemporal and corticobasal degenerations and PSP. Neurology 2006;66:41-48.

6. Ling H, O'Sullivan SS, Holton JL, et al. Does corticobasal degeneration exist? A clinicopathological re-evaluation. Brain 2010;133:2045-2057.

7. Haug A, Boyer P, Kluger B. Diffuse Lewy body disease presenting as corticobasal syndrome and progressive supranuclear palsy syndrome. Mov Disord 2013;28: 1153-1155.

8. Horoupian DS, Wasserstein PH. Alzheimer's disease pathology in motor cortex in dementia with Lewy bodies clinically mimicking corticobasal degeneration. Acta Neuropathol 1999;98:317-322.

9. Dickson DW. Dementia with Lewy bodies: neuropathology. J Geriatr Psychiatry Neurol 2002;15:210-216.

10. Montine TJ, Phelps CH, Beach TG, et al. National Institute on Aging-Alzheimer's Association guidelines for the neuropathologic assessment of Alzheimer's disease: a practical approach. Acta Neuropathol 2012;123:1-11.

11. Ferman TJ, Boeve BF, Smith GE, et al. Inclusion of RBD improves the diagnostic classification of dementia with Lewy bodies. Neurology 2011;77:875-882.

12. Fujishiro H, Ferman TJ, Boeve BF, et al. Validation of the neuropathologic criteria of the third consortium for dementia with Lewy bodies for prospectively diagnosed cases. J Neuropathol Exp Neurol 2008;67:649-656.

13. McKeith IG, Boeve BF, Dickson DW, et al. Diagnosis and management of dementia with Lewy bodies: fourth consensus report of the DLB Consortium. Neurology 2017; 89:88-100.

14. Braak H, Braak E. Neuropathological stageing of Alzheimer-related changes. Acta Neuropathol 1991;82:239-259.

15. Thal DR, Rub U, Orantes M, Braak H. Phases of A $\beta$-deposition in the human brain and its relevance for the development of AD. Neurology 2002;58:1791-1800.

16. Beach TG, White CL, Hamilton RL, et al. Evaluation of alpha-synuclein immunohistochemical methods used by invited experts. Acta Neuropathol 2008;116: 277-288.

17. Frigerio R, Fujishiro H, Ahn TB, et al. Incidental Lewy body disease: do some cases represent a preclinical stage of dementia with Lewy bodies? Neurobiol Aging 2011;32: 857-863.

18. Dugger BN, Murray ME, Boeve BF, et al. Neuropathological analysis of brainstem cholinergic and catecholaminergic nuclei in relation to REM sleep behaviour disorder. Neuropathol Appl Neurobiol 2011;38:142-152.

19. Murray ME, Graff-Radford NR, Ross OA, Petersen RC, Duara R, Dickson DW Neuropathologically defined subtypes of Alzheimer's disease with distinct clinical characteristics: a retrospective study. Lancet Neurol 2011;10:785-796.

20. Josephs KA, Whitwell JL, Boeve BF, et al. Anatomical differences between CBS corticobasal degeneration and CBS-Alzheimer's disease. Mov Disord 2010;25: $1246-1252$.

21. Dickson DW, Ahmed Z, Algom AA, Tsuboi Y, Josephs KA. Neuropathology of variants of progressive supranuclear palsy. Curr Opin Neurol 2010;23:394-400.

22. Josephs KA, Katsuse O, Beccano-Kelly DA, et al. Atypical progressive supranuclear palsy with corticospinal tract degeneration. J Neuropathol Exp Neurol 2006;65:396-405.

23. Wenning GK, Litvan I, Jankovic J, et al. Natural history and survival of 14 patients with corticobasal degeneration confirmed at postmortem examination. J Neurol Neurosurg Psychiatry 1998;64:184-189.

24. Constantinescu R, Richard I, Kurlan R. Levodopa responsiveness in disorders with parkinsonism: a review of the literature. Mov Disord 2007;22:2141-2148.

25. Josephs KA, Dickson DW. Diagnostic accuracy of progressive supranuclear palsy in the Society for Progressive Supranuclear Palsy brain bank. Mov Disord 2003;18 $1018-1026$. 
Kouri N, Murray ME, Hassan A, et al. Neuropathological features of corticobasal degeneration presenting as corticobasal syndrome or Richardson syndrome. Brain 2011;134:3264-3275

27. Dugger BN, Boeve BF, Murray ME, et al. Rapid eye movement sleep behavior disorder and subtypes in autopsy-confirmed dementia with Lewy bodies. Mov Disord 2012;27:72-78.

28. Sha SJ, Ghosh PM, Lee SE, et al. Predicting amyloid status in corticobasal syndrome using modified clinical criteria, magnetic resonance imaging and fluorodeoxyglucose positron emission tomography. Alzheimers Res Ther 2015;7:8.

29. Gomperts SN, Rentz DM, Moran E, et al. Imaging amyloid deposition in Lewy body diseases. Neurology 2008;71:903-910.

30. Cho H, Baek MS, Choi JY, et al. 18F-AV-1451 binds to motor-related subcortical gray and white matter in corticobasal syndrome. Neurology 2017;89:1170-1178.
31. Josephs KA, Whitwell JL, Tacik P, et al. [18F]AV-1451 tau-PET uptake does correlate with quantitatively measured 4R-tau burden in autopsy-confirmed corticobasal degeneration. Acta Neuropathol 2016;132:931-933.

32. Smith R, Schöll M, Widner H, et al. In vivo retention of 18F-AV-1451 in corticobasal syndrome. Neurology 2017;89:845-853.

33. Heilman KM, Gonzalez Rothi LJ. Apraxia. In: Heilman KM, Valenstein E, editors. Clinical Neuropsychology, 5th ed. Oxford, UK: Oxford University Press; 2012: 214-237.

34. Boelmans K, Kaufmann J, Bodammer N, Heinze HJ, Niehaus L. Corticospinal tract atrophy in corticobasal degeneration. Arch Neurol 2006;63:462-463.

35. Braak H, Rub U, Schultz C, Del Tredici K. Vulnerability of cortical neurons to Alzheimer's and Parkinson's diseases. J Alzheimers Dis 2006;9(3 suppl): $35-44$. 